\title{
Simulation of traffic management with FRISO
}

\author{
A. D. Middelkoop ${ }^{1}$ \& L. Loeve ${ }^{2}$ \\ ${ }^{1}$ ProRail Strategy and Innovation, The Netherlands \\ ${ }^{2}$ Incontrol Enterprise Dynamics, The Netherlands
}

\begin{abstract}
Due to the high occupancy rate of the Dutch rail network it is difficult to handle future transport demand by the introduction of more trains. The main challenges are on one hand generating a feasible timetable and at the same time meeting performance criteria e.g. punctuality and train delays. Possible solutions are extension of infrastructure capacity and/or adjustment of the timetable structure. Besides those more common measures there is a third option that focuses on traffic management. These measures concern operational and planning issues such as increasing the level of flexibility in daily operation, e.g. by better decision support systems, and decreasing the level of detail in the planning stage. In order to quantify the improvements of such measures, Dutch Inframanager ProRail uses simulation. This simulation approach requires new functionality, regarding train control mechanisms.

One of the first studies performed with this kind of simulation is the important bottleneck Schiphol station. Following this approach the development of a more general simulation tool has been started. This tool is called FRISO: Flexible Rail Infrastructure Simulation of Operations. Important features of this tool are the automatized construction of a simulation model by using a connection to an existing infrastructure database, flexible infrastructure editor including generator functions and the possibility to perform single and multiple (stochastic) simulation experiments.

A special feature is the possibility to connect to an existing external traffic management system (TMS). By this connection the effects of TMS-variants may be quantified. The software architecture contains synchronisation mechanisms that allow consistent time management and interaction between the simulator FRISO and TMS or other applications. TMS can take over internal FRISO train dispatching tasks to optimise the train traffic in a local area. It is designed to improve traffic control by means of advisory speeds, routes and order of trains. Keywords: simulation, traffic management, distributed simulation.
\end{abstract}




\section{Introduction}

This paper describes the development of a new train simulator called FRISO, which stands for Flexible Rail Infra Simulation of Operations. After a short introduction of the Dutch Rail Network in section 2, it explains the programme of Dynamic Traffic Management (DTM) in section 3. This programme consists of a number of measures which change the planning and the operation principles of the railway traffic. A particular part of this programme is the development and introduction of a Traffic Management System (TMS) which optimises traffic performance on punctuality, energy consumption and throughput.

Next, section 4 describes the simulation approach to study the effects of DTM, starting with the first experiments/studies regarding Schiphol, the railway station of Amsterdam Airport. Then follows a more general approach, which requires a flexible simulation tool (FRISO) for investigating traffic control solutions for potential bottlenecks in an efficient way. Then section 5 explains a special feature of the approach, the connection of TMS and FRISO as part of a High Level Architecture (HLA), which enables consistent synchronisation of so called simulation federates and flexible exchange of information and functionality/software.

The paper ends with some remarks on current status and following activities.

\section{The Dutch Rail network}

The size of the infrastructure of the Dutch railway network is moderate compared to most other European countries. The length is $2800 \mathrm{~km}$ and it contains about $6500 \mathrm{~km}$ of tracks. Extension of the network is foreseen to be ready in 2007. Then a new high speed line Amsterdam-Schiphol-RotterdamBelgium, a new connection between the harbour of Rotterdam and Germany (Betuweroute) and a new extra double track connection between Amsterdam and Utrecht will be available.

The main part of the traffic (128 million trainkm in 2002) is used for passenger transport. On a daily basis some 5000 trains carry 1 million passengers. Freight transport is growing more important, but is still a minor part (about $8 \%$ of the trainkm performance). Each day about 280 cargo trains transport 80 kton of freight.

The occupation rate of the Dutch rail network is high [1.]. This is illustrated in figure 1. In this graph the trainkm/netkilometer $(\mathrm{x} 1000)$ rate per year for a number of countries is shown.

The actual state of the railway-infrastructure and the expected growth of passengers- and freight traffic don't match. The current timetable structure cannot transport these amounts of passengers and freight, so more trains are necessary. The introduction of more trains causes not only capacity problems in the network, but also a decrease of the performance, measured by punctuality and train delays. Insufficient capacity on potential bottlenecks may be solved by extending the infrastructure capacity. However, building railway infrastructure is very expensive and it takes a long time to design and fit it into the environment. 
So new and innovative measures are necessary to be able to operate an attractive and cost-efficient timetable. One of those measures, a programme called Dynamic Traffic Management, is explained in the next section.

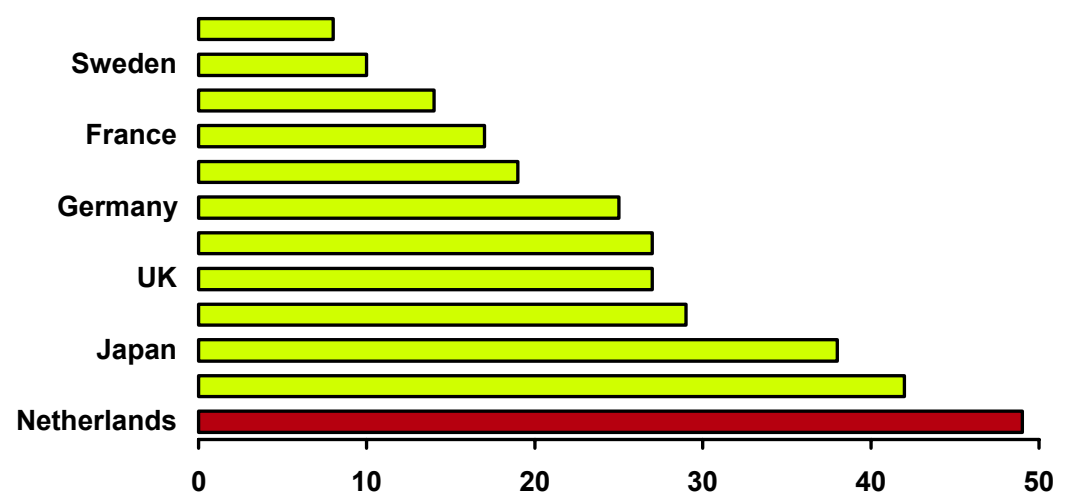

Figure 1: Occupation rate: x1000 trainkm/netkm per year in 2002.

\section{Dynamic Traffic Management}

At ProRail, the Dutch rail infrastructure manager, there is a programme of new innovative ideas which investigates the introduction of Dynamic Traffic Management (DTM). Main issue of DTM is to accept the fact that in daily operation deviations of the planned situation will occur. The aim is to manage these deviations effectively the moment they take place to improve the performance. The programme consists of measures that change the design and operation of the timetable by introducing innovative planning principles and advanced new control strategies. Problems are not statically avoided in the planning, but dynamically solved in real time. To predict the effect of those measures on timetable quality ProRail uses a simulation approach.

\subsection{Changing planning principles}

Railway transport has a lower level of operational freedom than other transport modes. Railway companies plan the train services in a very detailed way. The train order at crossings, junctions and platform tracks is fixed more than a year in advance to prevent conflicts and to check the capacity. In practice trains rarely travel exactly to the plan as illustrated in figure 2 . In the right part of the figure each line represents the running of a train on a certain day in March 2003. The plan contains fixed recovery times to handle daily variations. The DTM concept aims on a flexible use of these recovery times. Thus the main focus is not on planning but on performance of the train services.

The DTM concept puts the reliability first. An organic planning approach takes into account the daily variation performed in practice. The required running 
and dwelling times as demonstrated in practice serve as the starting point for constructing a reliable timetable. It determines how much margin time is needed to obtain a certain level of reliability. This feedback can support both the short term operational process as well as the long term planning process.

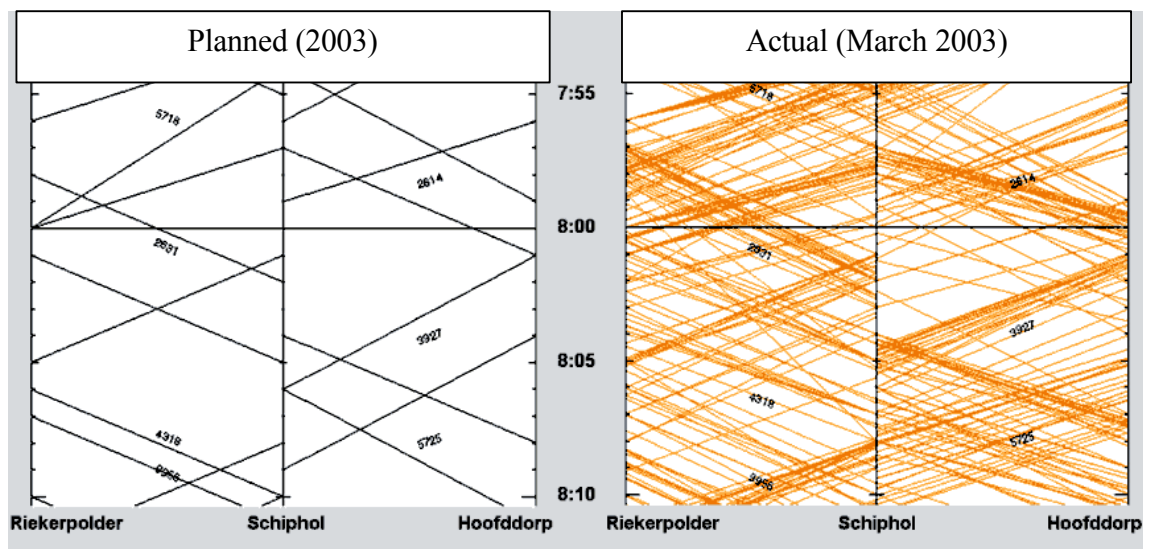

Figure 2: Planned and actual use of the train services around Schiphol station during march 2003 (source: ProRail)

Once we accept that disturbances are part of our reality, we need the flexibility to handle them. Therefore we must avoid certain details in our planning. This can be done in different ways: time windows, dynamic use of tracks, dynamic change of order. Flexible planning places a train in a time window (no exact time) and plans it to arrive at a certain range of platform tracks. The precise platform track is decided (and communicated) during operation, as the actual traffic situation is known. The earliest departure time and the latest arrival time are published as a customer timetable. The first bottleneck investigated in this way is described in section 4.1.

Flexible planning also enables an exchange of buffer times. In bottlenecks buffer times are no longer fixed between trains. Some conflicting train movements are thus allowed in the planning. In practice the conflict might not even occur, or a solution will be found. The best order of train movements is chosen real time. This assumes a very good traffic control including powerful decision support systems.

\subsection{Supporting the traffic control}

As the planning is less detailed the details may be managed in real time. Traffic control thus requires more real time actions. The dispatcher needs more support to perform this extended task. DTM defines two types of support: simple and advanced traffic control.

Simple traffic control uses predefined sets of dispatching rules, based on offline analysis. Advanced traffic control uses real time control, based on on-line 
analysis. Both approaches need support systems either to improve decisions or to automatically perform (parts of) the dispatching task.

Local systems act only "on the spot" and "now". They may act according to dispatching rules such as "First Come First Served". In some situations the rules must be extended with detailed local dispatching rules.

Advanced traffic management systems take into account all traffic in a certain area. They predict conflicting train movements, calculate an optimal traffic flow and present advisory speeds to train drivers. They also advise a change of order or route to the dispatcher. In the COMBINE research project [2] a demonstrator has shown the effects of such a real-time traffic management system (TMS). It optimises the traffic performance on punctuality, energy consumption and/or throughput. In the Netherlands a prototype has been tested in operation. The results were promising, punctuality is expected to rise about $3 \%$, the energy consumption about $7 \%$. This is reason to study the effects of the TMS on other potential bottlenecks. To prevent high costs of testing in reality and redevelopment of functionality, the TMS therefore should be connected to a simulator.

This requires a very flexible simulation environment, which must be able to handle different traffic control strategies, perform stochastic simulation experiments and connect easily to different versions of traffic management systems.

To enable future exchange of new components and already existing functionality both the simulator and the TMS will be part of a High Level Architecture (HLA). This HLA [3] supports interoperability between different simulation models and enables the reuse of existing functionality and software. This is further elaborated in section 4.2.

\section{Simulation approach}

To predict the change in the performance of a timetable, as a result of the introduction of simple or advanced DTM-measures, a simulation approach is required. One of the first bottlenecks in the network where DTM-solutions are necessary is the railway station of Amsterdam Airport, Schiphol. Section 4.1 describes a simulation study, where this approach is applied, supported by a prototype version of a simulator. The results were reason to investigate further introduction of DTM-principles and to start the development of a generic flexible simulation tool called FRISO. This tool is described in section 4.2.

\subsection{Schiphol simulation study}

The railway station of Amsterdam Airport, called Schiphol, is situated underneath the entrance hall of the airport, so travellers can enter and leave the airport by train very easily. The layout of the station is shown in figure 3 .

Today 14 trains per hour run through the tunnel, but in less than 4 years time this should be almost doubled. Given the current rail infrastructure and the origin and destinations of the train services, the number of conflicting situations 
between trains is expected to increase rapidly, which will affect train delays and punctuality. Adding tracks is extremely expensive due to the underground location and changes to the timetable do not seem to be sufficient, so other measures are necessary.

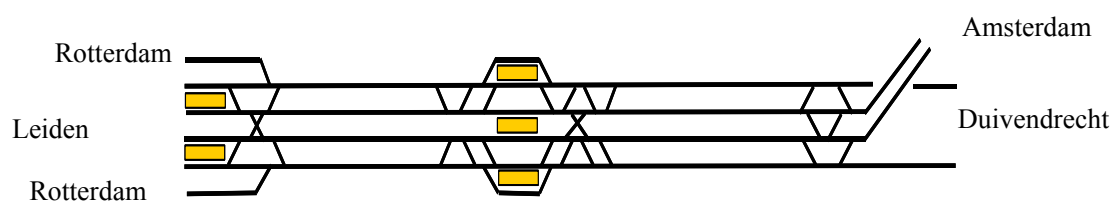

Figure 3: Layout infrastructure Schiphol.

Therefore combinations of small changes to the infrastructure and DTM-solutions were evaluated. The first proposed solution concerns decreasing the minimum time between subsequent trains. By removing some switches, it is possible to shift signals towards the platform. In this way, it takes less time for waiting trains to enter the platform. A second solution for decreasing the minimum time between trains is to decrease the setting times of signals significantly.

As a third solution based on DTM, introduces time windows into the timetable. Trains can leave stations earlier due to the less stringent departure times, thus optimising dwelling times. The introduction of a control strategy, based on the First Come First Served principle is a fourth option. The order of trains as given in the plan is not preserved but based on the actual arrival times of trains at a controlled area. As final solution, the dynamic use of platform at Schiphol is proposed, under the condition that passengers should be informed in time of platform changes.

The above mentioned solutions were combined in several scenarios. To determine the impact of the solutions on resulting running times simulation was used. Especially for the Schiphol area a prototype of a flexible simulation tool was implemented. This prototype generates simulation models out of existing databases and is designed in such a way that it is very easy to change control strategies, setting times of signals and platform usage. As performance indicator the 87 percentile of running times was measured. The number of trains per hour was increased at each experiment, resulting in longer running times. The current planned running times of trains in the study area was compared with the experiment results.

The simulation study showed that without changes in infrastructure, the desired amount of trains is possible when planned running times are extended with 3 minutes. Changes in infrastructure have a positive effect on the capacity, but not enough to handle the desired amount of trains with the current planned running times. DTM-measures are therefore necessary. The most effective scenario contained first come first served principles together with dynamic platform usage. However, to satisfy a desired punctuality of $87 \% 1$ minute extra running time is needed in the timetable. The results were so promising that at the 
end of 2005 a real life test was performed at Schiphol, showing that the simulated control strategies are also feasible in practice.

The results were reason to test DTM-principles in reality as a pilot project and to investigate introduction of both simple and advanced DTM-principles on other bottlenecks. Necessary further development of the simulation prototype to a flexible tool is described in the next section.

\subsection{FRISO: Flexible Rail Infra Simulation of Operations}

Performing a simulation study is very powerful but has also some disadvantages. These are related to the time effort needed, to the software architecture and therefore the costs.

Looking at the time needed for a simulation analysis, a great part of it is used for building (often manually) and validating the simulation model of a situation. The investigation of a new situation causes an equal development effort. Here a generation of the simulation model based on input from company databases, in combination with smart editors for making timetable and infrastructure variants, can reduce the time effort significantly.

Often railway simulators are tailor made for a certain railway system. They originate from the time that object oriented programming was not yet a standard. This makes it difficult and expensive to introduce properties of new other railway systems or to change existing properties, e.g. regarding the way the signalling is modeled.

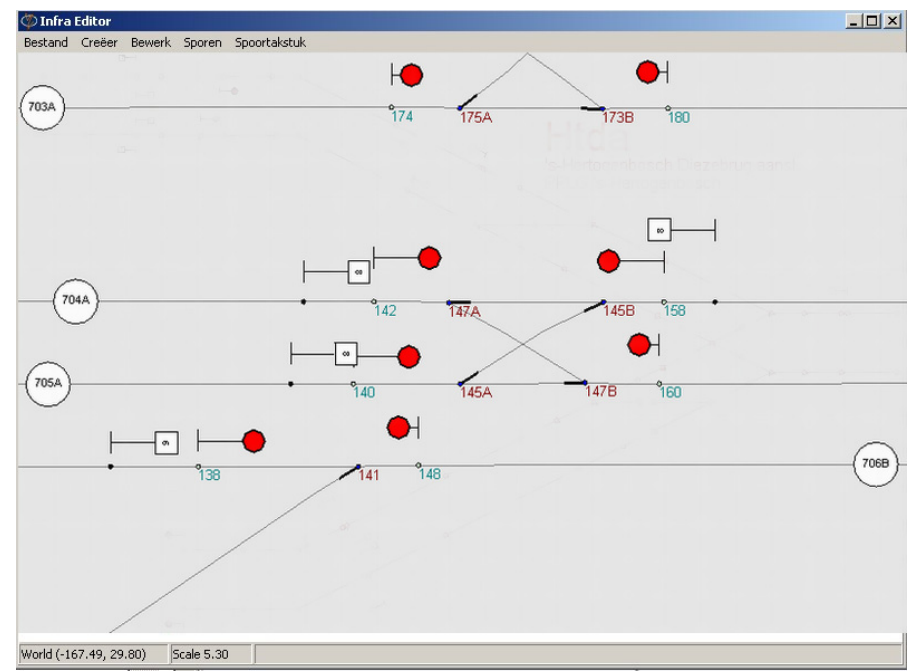

Figure 4: $\quad$ FRISO infrastructure editor.

With the development of FRISO, ProRail adopted the approach of SIMONE, a countrywide network simulator [4], to overcome these disadvantages. Both tools are based on a general simulation language/tool called Enterprise 
Dynamics, which is used in a lot of industries. On top of the simulation engine ProRail developed a library of railway components. A simulation model may be generated automatically by 'filling' the components with timetable and infrastructure data. The user can spend most of his time on the definition of simulation experiments and the analysis of the results.

FRISO models the following elements of the railway infrastructure: track layout, signalling system, route setting, and interlocking, see figure 4 . In the timetable trains are allocated to routes. The data is imported from timetable an infrastructure databases (Infra Atlas). To make simulation variants FRISO has a number of edit functions, to change infrastructure, timetable and traffic control options. These functions will be enhanced in future versions with smart generators for signalling and interlocking elements and route definition.

A special feature of the flexibility is the possibility to connect to other applications that may take over parts of the functionality. A first example, the connection between an advanced traffic management system and FRISO, is explained in section 5 .

\section{FRISO and TMS}

A train simulator has functionality, mostly a set of rules, to control the train traffic. For testing the TMS benefits these rules become partly redundant. The idea is that the TMS will improve the traffic performance by optimising the situation on punctuality, energy consumption and/or throughput. It therefore must be able to control the trains and exchange information on the actual position and status of the trains with the simulator. Connecting existing functionality as the TMS to the simulator is a form of distributed simulation. The simulation scenario will contain several components, so there is a need for a control mechanism. Especially the time management is an important issue. The FRISO-TMS combination uses an architecture which enables interoperability of simulation models, the High Level Architecture (HLA) [3]. This standard promotes re-use of (different kinds of) simulation models and their components. It specifies interfaces between components and defines the steps for the development and execution of a simulation scenario. The applications in such a scenario are also known as federates. They form a federation and have to cooperate, communicate and synchronize mutual information. In the simulation FRISO informs the TMS about location and status of the trains, occupation of the infrastructure, allowed speed profile restricted by the signalling system and allocation of routes. The TMS returns advisory speeds, booking of routes and (later also) change of track allocation.

The architecture of an HLA-connection between TMS and FRISO is shown in figure 5. To translate messages of FRISO and TMS to HLA, mappers are used. These are connected to the run time infrastructure based on HLA principles. HLA takes care of time synchronization, saving and restoring simulations and updating information. By using the mappers and HLA it becomes very easy to replace the TMS with another traffic management system or to replace FRISO 
with real life trains. The only modification needed then is to implement a mapper for the newly connected system, translating messages into HLA-messages.

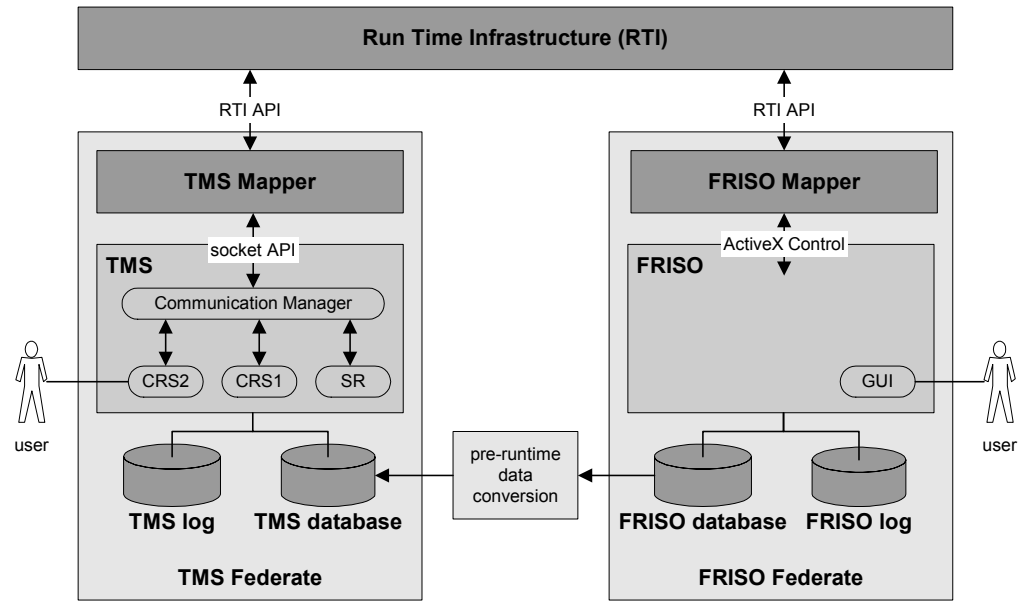

Figure 5: $\quad$ Architecture FRISO and TMS.

\section{Future research}

The current state of the development is that the first version of the simulator FRISO is validated on a part of the network. The next steps of the development will increase the flexibility of the tool by improving the user interface and by introducing more input generators.

The connection with the TMS is ready. It will be used in a series of simulation experiments to investigate the DTM-effects and the tuning of the TMS on several junctions. First results will be available in June 2006.

\section{References}

[1] Poort, J.P.: Limits on utilization (in Dutch). NYFER (2002)

[2] De Vries, D.: COMBINE a decision support system for real time traffic control. Computers in Railways VIII (2002) 1003-1010

[3] IEEE Standards 1516.3: Recommended practice for High Level Architecture (HLA), Federation Development and Execution Process (FEDEP). Institute for Electric and Electronic Engineers (2003)

[4] Bouwman, M., Middelkoop, A.D.: Testing the stability of the rail network. Computers in Railways VIII (2002) 995-1002 